\title{
Impact of Constraint Conditions and Cutouts on Natural Frequency of Glass Fibre Reinforced Plastic Composite
}

\author{
Madhav Murthy, Kayala Mallikharjuna Babu and Peter Martin Jebaraj
}

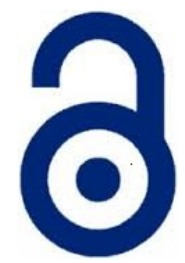

Received: 12 September 2018

Accepted: 03 November 2018

Published: 01 December 2018

Publisher: Deer Hill Publications

(c) 2018 The Author(s)

Creative Commons: CC BY 4.0

\begin{abstract}
Majority of the activities of a human being is associated with vibrations of one or other form. Application of vibrations can be found from a simple example like listening to music to challenging ones like design of complex machines and structures. Design of any system will not be rugged if it does not consider vibrations and its effects. The energy dissipated per cycle can be understood as damping. Presence of damping in a structure is represented by damping factor or damping ratio. Fibre reinforced plastic composites are a class of materials made from matrix and reinforcement. Matrix can be a resin system and reinforcement is usually fabric made of glass, carbon or aramid. The vibrating mechanical system causes resonance resulting in failure of machine components. By knowing the natural frequency and changing it from the range of operating machine member, resonance phenomenon could be avoided. This work focuses on finding the damping ratio and natural frequency of $s$ glass fibre reinforced polymer composites through harmonic \& modal analysis both by experimental method and FEM analysis for bare plate, plate with rectangular cutouts and plate with circular hole. The reduction in natural frequency and increase in damping corresponds to a change in stiffness and damping of the system. These changes are attributed to freeness of the system and introduction of cutouts in the laminate.
\end{abstract}

Keywords. Vibrations, Damping factor, glass fibre reinforced composites, natural frequency

\section{INTRODUCTION}

Man became interested in vibration when he created the musical instruments like drums, whistles and other musical instruments. Since then, philosophers and musicians came up with the understanding of sound and sounds, which were used in creation of various musical instruments and were passed from generation to generation [1]. The motion that repeats itself after an interval of time can be called oscillation or vibration. The swinging of a simple pendulum, motion of a tuning fork and the spring balance are typical examples of vibration. Many a human activities involve sound and vibration in one or the other form. The theory of vibration deals with the study of oscillatory motion of bodies and forces associated with them. Vibration can be desirable or undesirable. Sound and vibration studies are interrelated. The types of vibration are free, forced, damped and undammed.

\subsection{Loss Factor}

When a structure is excited, the vibrations in the structure do not last forever and it therefore dampens out. The ability of structures, which dissipates vibrational energy, is an important parameter of a system called damping which

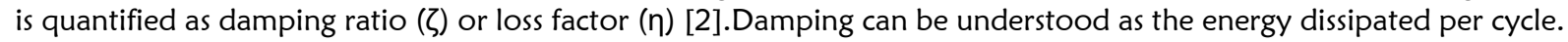
When there is a presence of damping in a structure, it is generally represented by damping factor or damping ratio and when it is defined for a specific mode, it is known as modal damping factor.

A structure can have many natural frequencies. For a design to be reliable, estimation of damping becomes important. Loss factor estimation is straightforward unlike other parameters like stiffness and mass. It is often evaluated during experimentation. Inconsideration of loss factor may not lead to a sound design. Hence, a suitable damping is to be provided for increasing the weight, whereas overestimation of loss factor may lead to unwanted

\footnotetext{
M. Murthy $\bowtie$, K. M. Babu and P. M. Jebaraj

Department of Mechanical Engineering

B.M.S. College of Engineering

Bull Temple Road, Bengaluru

Karnataka, India-560019

E-mail: madhavmurthy.mech@bmsce.ac.in
}

Reference: Murthy, M., Babu, K. M. and Jebaraj, P. M. (2018). Impact of Constraint Conditions and Cutouts on Natural Frequency of Glass Fibre Reinforced Plastic Composite. International Journal of Engineering Materials and Manufacture, 3(4), 200-207. 
vibrations and sometimes breakdown of the structure prior to the designed life. Some of the common issues associated with loss factor estimation are to understand the limits and requirements in each method of loss factor estimation. It is crucial, as there is no single method suited for all situations [3]. Most techniques do not accurately estimate high loss factors, mostly because of insufficient/incorrectness of data provided. While taking measurements, the positioning of excitation point and sensors is vital since multiple input-output locations of the structure are involved. A structure should never be excited at a node line as this may cause rigid body response. It is also important not to measure response from any point on node line $[4,5]$. For simulating a continuous system efficiently and removing spatial effect from measurements, long sets of time history of responses needs to be recorded and analysed from multiple locations, which best be randomly chosen. In cases where multiple input and output locations are involved, slope fitting can prove to be another challenge. When a great number of slope fits are required to be performed, human error is unavoidable which may lead to inaccuracy of results. Therefore, an automated slope fitting algorithm is recommended. To avoid any probable biased error, visual inspection helps to understand the decay curves for all slope fits [6].

\subsection{Half Power Bandwidth Method}

It is a frequency domain method used for estimation of loss factor. The estimation of loss factor is carried out using frequency response functions. The expression of damping factor is given by $\zeta=\left(\omega_{n 2}-\omega_{n 1}\right) / 2 \omega_{n}$ where $\omega_{n}$ is the natural frequency, $\omega_{n 2}, \omega_{n 1}$ are half power points and the difference of these is called the bandwidth. The objective of this work is to estimate the damping factor of glass fibre reinforced composite plates (laminates) with no cutouts, circular cutouts and rectangular cutouts and varying boundary conditions [7, 8]. The boundary conditions considered in this work are clamped-clamped-clamped-clamped (CCCC), clamped-clamped-clamped-fixed (CCCF) and clamped-fixed -clamped-fixed (CFCF). The laminates were fabricated using resin transfer moulding (RTM) method. The matrix was the combination of resin and hardener in the ratio of 1:10. The matrix and reinforcement used in this study are Epoxy LY556 with HY951 as hardener and bidirectional S glass fibre as the reinforcement. The laminate was cured at room temperature. The machining of the laminates was carried out in abrasive water jet machining centre $[9,10]$.

The validation of experimental and FEA values of damping factor and natural frequency results were carried out. Fast Fourier transforms (FFT's) were obtained from the experimental studies. The boundary conditions used for experiments to determine natural frequencies and FEA were the same. There was a variation between the FEA and experimental results (analytical vs experimental) which could be due to the mesh quality, section of the element and accuracy of boundary conditions, which should imitate the experimentation condition for validation of FEA results. All the plates used for experimentation are fabricated using $S$ glass fibres with the epoxy resin LY556 and the hardener HY951. The composite laminate was fabricated by resin transfer moulding process. The plate dimensions were chosen as $420 \mathrm{~mm} \times 210 \mathrm{~mm} \times 2 \mathrm{~mm}$ for the final laminate size. The numbers of layers of reinforcements used are 8 in addition to resin contributing to the rest of thickness and resin-hardener in the ratio of 1:10. The workpiece and experimental setup is shown in Figure 1 and Figure 2 respectively. Figure $1 \mathrm{a}, 1 \mathrm{~b} \& 1 \mathrm{c}$ indicates the laminates fabricated and machined to the dimensions and $1 \mathrm{~b}-1 \mathrm{c}$ have rectangular and circular cutouts for testing the damping and six modes of frequency.

\section{METHODOLOGY}

\subsection{Experimental Procedure}

The measurement of vibration characteristics depends on various factors such as position of exciter to excite the structure, placement of an accelerometer, and clamping of the plate etc. The brief procedure for the experimentation is as follows.

The Plate is clamped firmly after the dimensions are measured for all the configurations (CCCC, CCCF \& CFCF) and Smearing of wax on the uniaxial accelerometer is carried out before placing the accelerometer on the vibrating structure. The structure is then excited by sine sweep method. Then the response of structure is recorded using data acquisition system and compatible software.

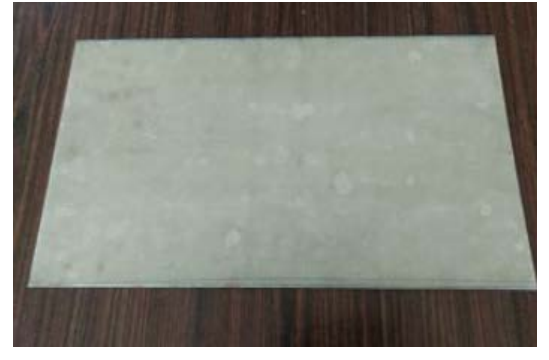

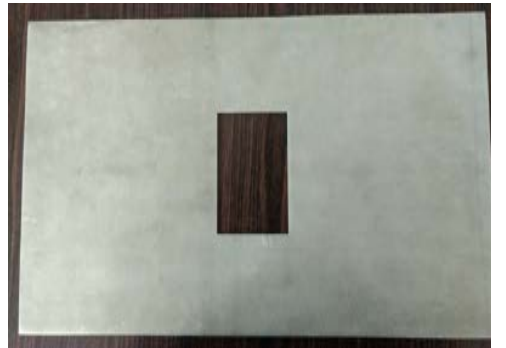

$\mathrm{b}$

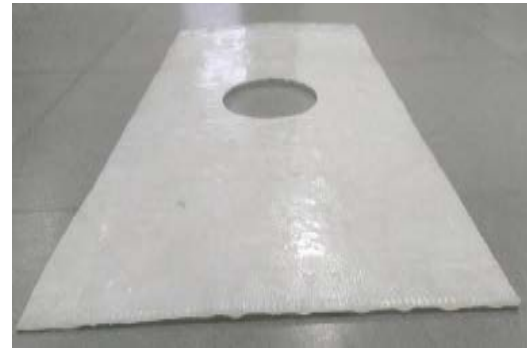

C

Figure 1:Tthe workpiece (a) bare plate, (b) late with rectangular cutout, and (c) plate with circular cutout After the data acquired it is exported to an FFT analyser and read in frequency domain, which shows amplitude response corresponding to frequency. The amplitude response is as shown in Figure 3 in Hertz. Natural frequencies at various frequencies and intervals of time can be seen in Figure 3. 


\subsection{Validation using FEM Approach}

The finite element analysis for the orthotropic laminates prepared with the above-mentioned boundary configurations is carried out. Frequency response curves obtained experimentally and through ANSYS is shown in the Figure 4. Quality factor is calculated for all the configurations using Half Bandwidth technique. In experimental method, frequency response curves are plotted as frequency $\mathrm{v} / \mathrm{s}$ amplitude. The amplitude obtained experimentally is shown in terms of volts, larger the volts larger is the amplitude. The plate (laminate) response is taken from the analyser and the accelerometer sends the signals to the analyser in the form of volts as can be seen in figure 4. Voltage is a direct measure of amplitude. In Finite Element Analysis, the frequency response curve, magnitude of the amplitude can be obtained. Highest peak in experimental frequency responses via FFT and FE frequency response curves from ANSYS corresponds to the natural frequency of the structure

The S Glass FRP laminates are used for the vibration analysis. With the fibre and resin composition of $60-40 \%$ respectively. The plates are subjected to loads such as fatigue, point loads, bending etc. so the plate should have the capability to resist such loads. The Table 1 shows the physical properties of the reinforcement. Modulus, density and thickness are important parameters for carrying out simulation and experimental studies, which are given in Table 1.

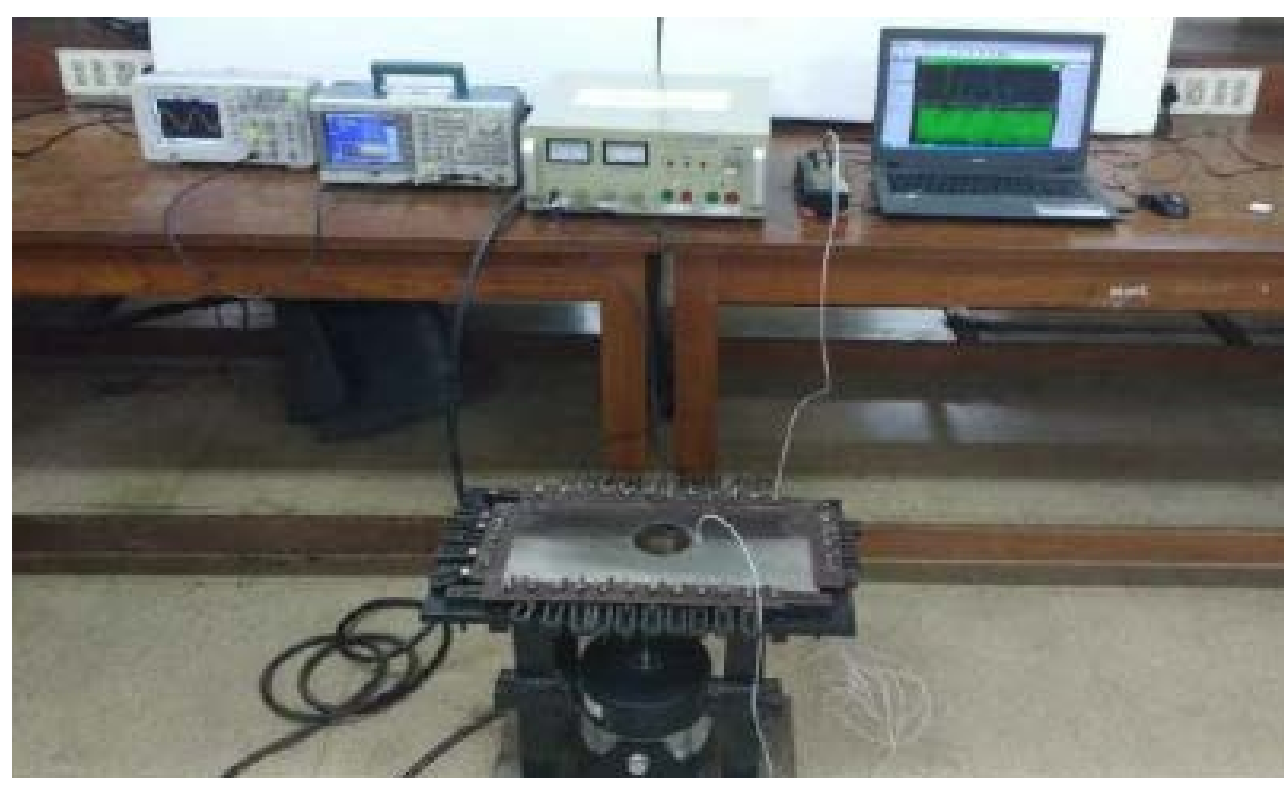

Figure 2: Experimental setup with the fixtures

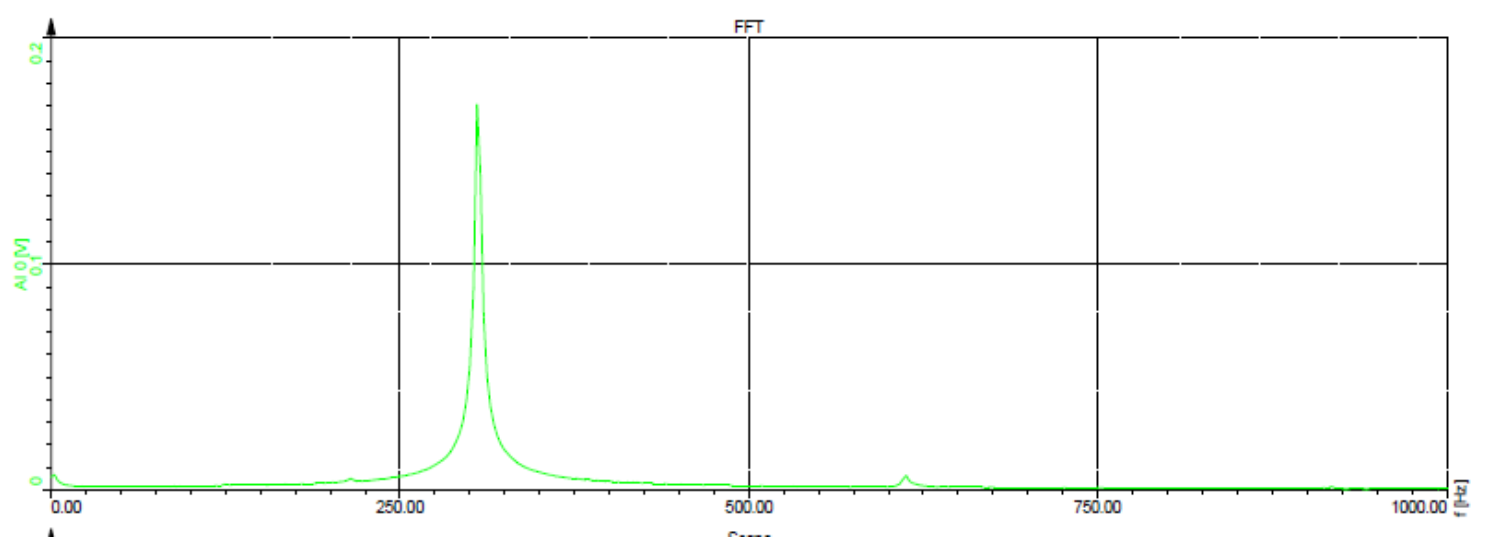

Figure 3: Amplitude response of bare plate with $\mathrm{CCCC}$ configuration $(\mathrm{Hz})$ 


\subsection{Boundary conditions}

The orthotropic laminate is studied for different boundary conditions. The clamping is made on the edges of the plate. These three boundary conditions are shown in Figure 5. The various boundary conditions studied are CCCC (all ends clamped) for a bare plate (i.e., without any cut out), a plate with circular hole or cutout and a plate with rectangular cutout for carrying out further analysis as can be seen in figure 5 . The finite element method analysis is considered and obtained for six mode shapes. In this article only the first mode shapes are discussed as shown in Figure 6, Figure 7 and Figure 8. Once the meshing is done and the constraints are defined the next step is to carry out the modal as well as harmonic analysis for the orthotropic plates by providing the inputs of the frequency and the obtained results is as shown in Figures 6-8. In Figure 6, Figure 7 and Figure 8 nodal solutions are obtained and further analysed.

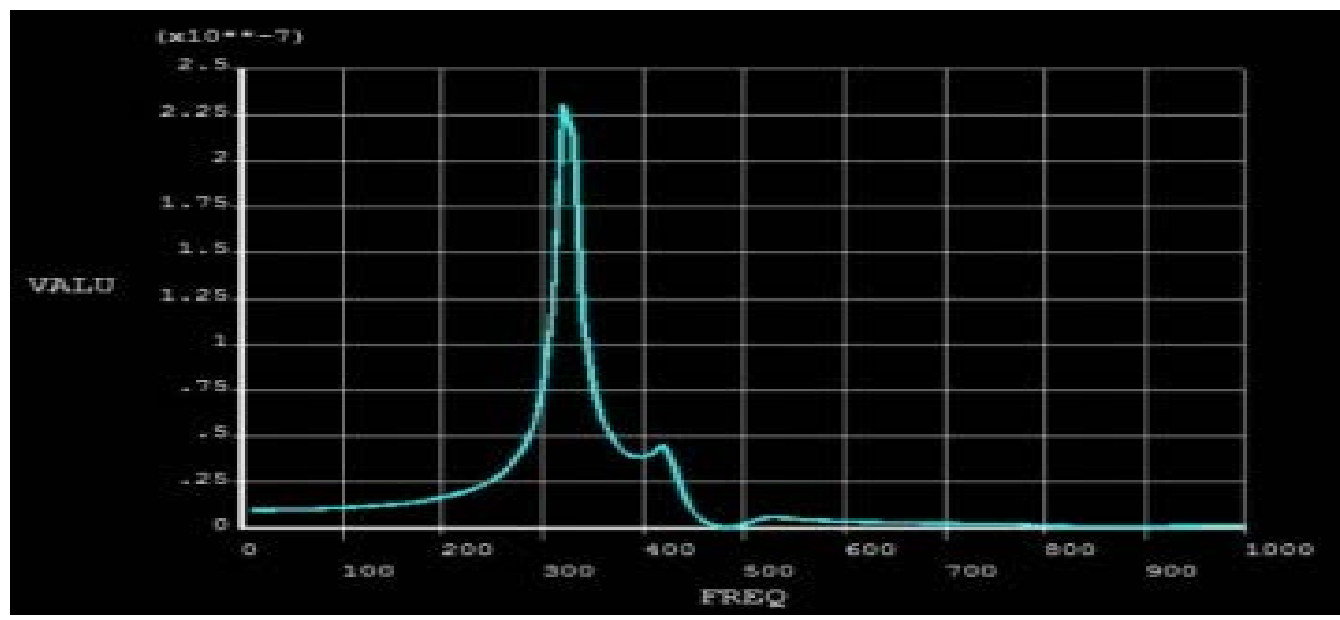

Figure 4: Plate with circular hole (CCCF condition)

Table 1: Properties of S Glass Bidirectional fabric

\begin{tabular}{ll}
\hline Properties & Values \\
\hline $\mathrm{E}_{1}$ & $85 \mathrm{GPa}$ \\
$\mathrm{E}_{2}$ & $3.5 \mathrm{GPa}$ \\
$\mathrm{E}_{3}$ & $3.5 \mathrm{GPa}$ \\
$\mathrm{V}_{12}$ & 0.2 \\
$\mathrm{~V}_{23}$ & 0.2 \\
$\mathrm{~V}_{31}$ & 0.35 \\
$\mathrm{G} 12$ & $36 \mathrm{GPa}$ \\
$\mathrm{G} 23$ & $36 \mathrm{GPa}$ \\
$\mathrm{G} 31$ & $36 \mathrm{GPa}$ \\
Density & $1822 \mathrm{~kg} / \mathrm{m}^{3}$ \\
Fabric thickness & $0.25 \mathrm{~mm}$ \\
\hline
\end{tabular}

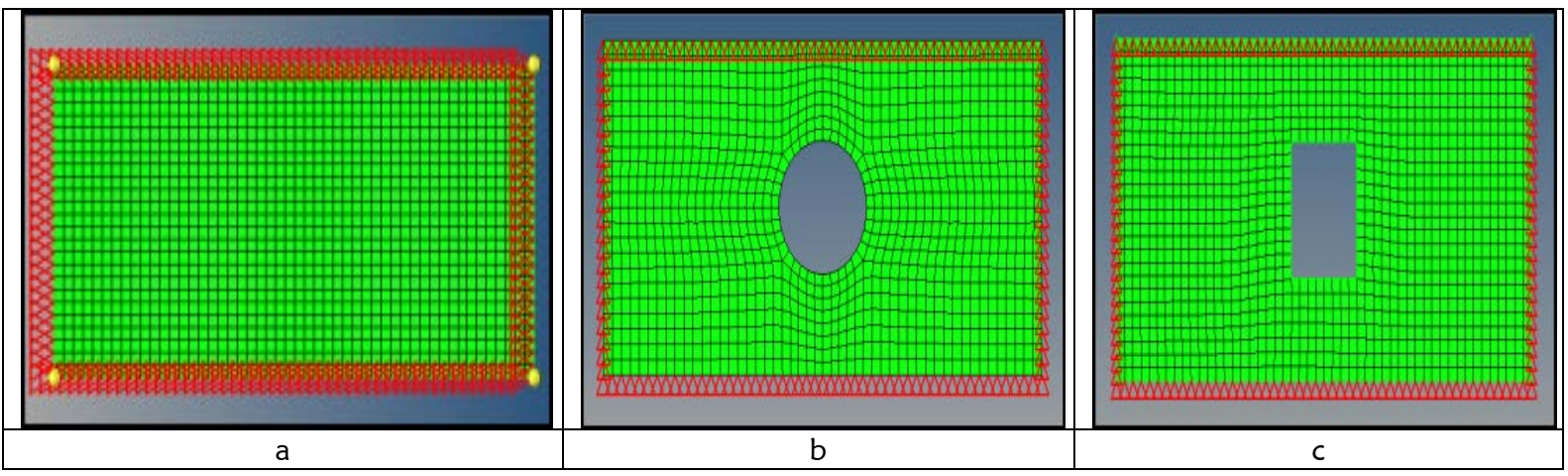

Figure 5: CCCC boundary configuration of (a) bare plate, (b) plate with circular hole and (c) plate with rectangular cutout. 


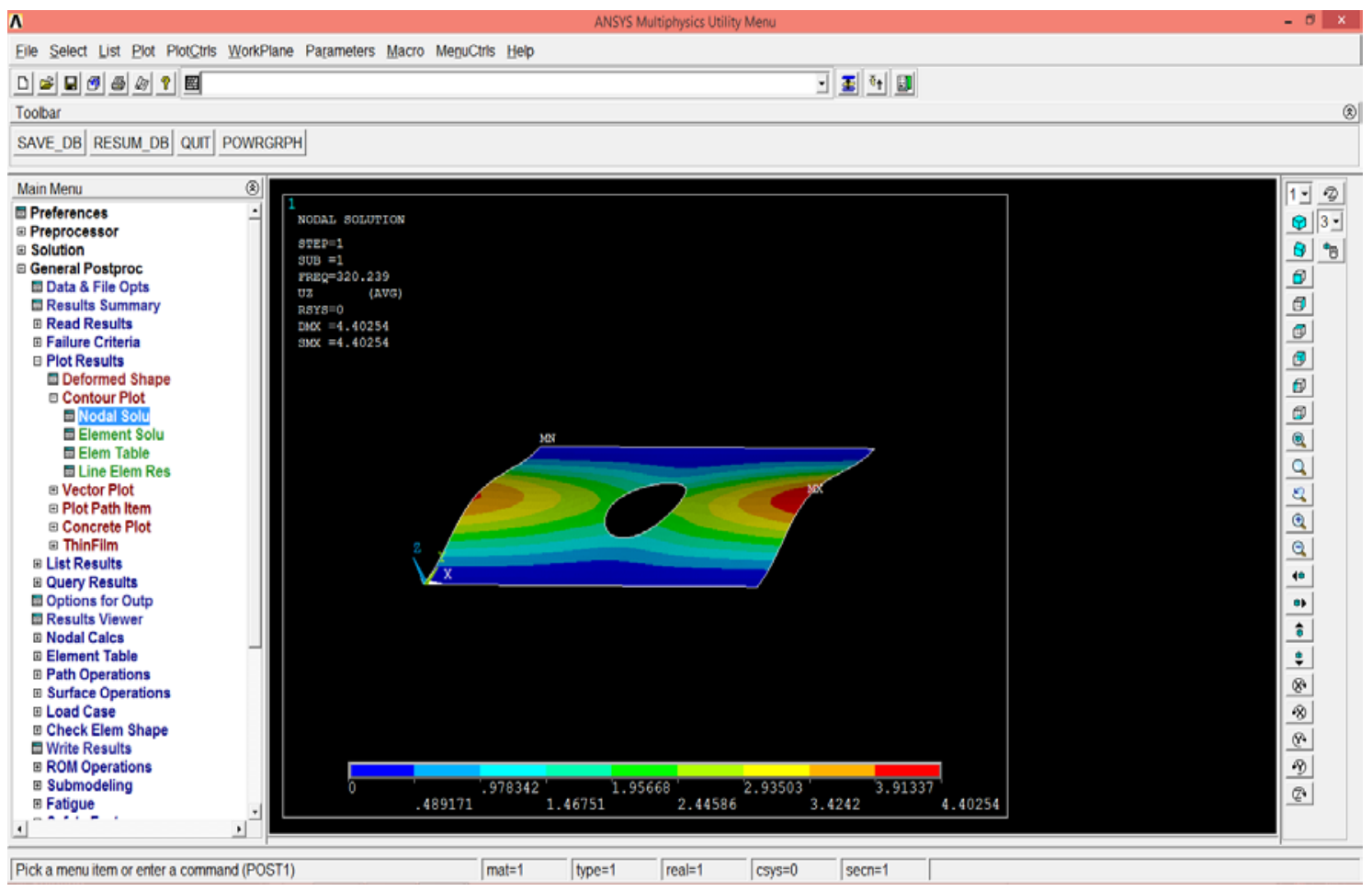

Figure 6: Plate with circular hole CFCF condition

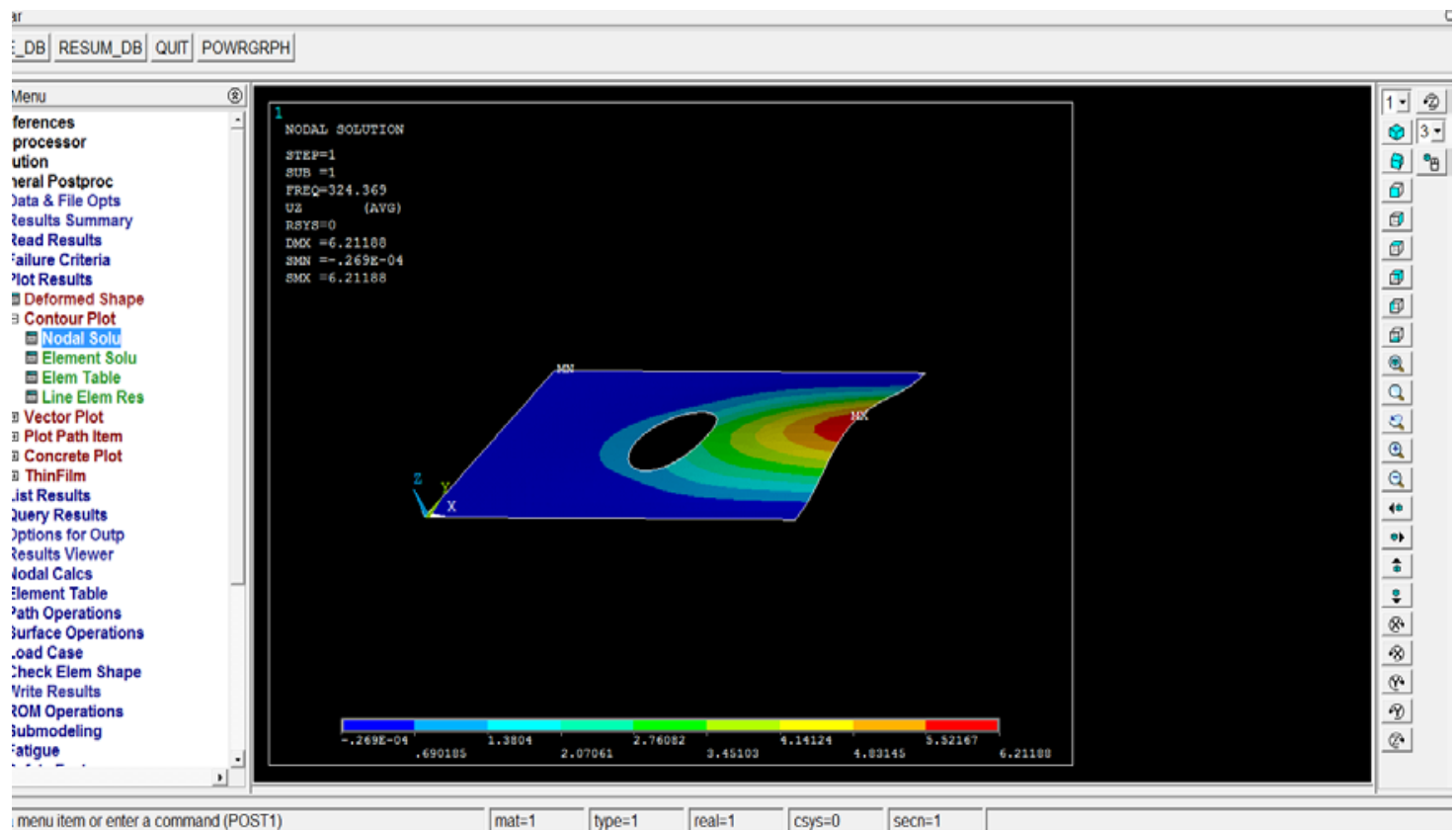

Figure 7: Plate with circular hole CCCF condition 


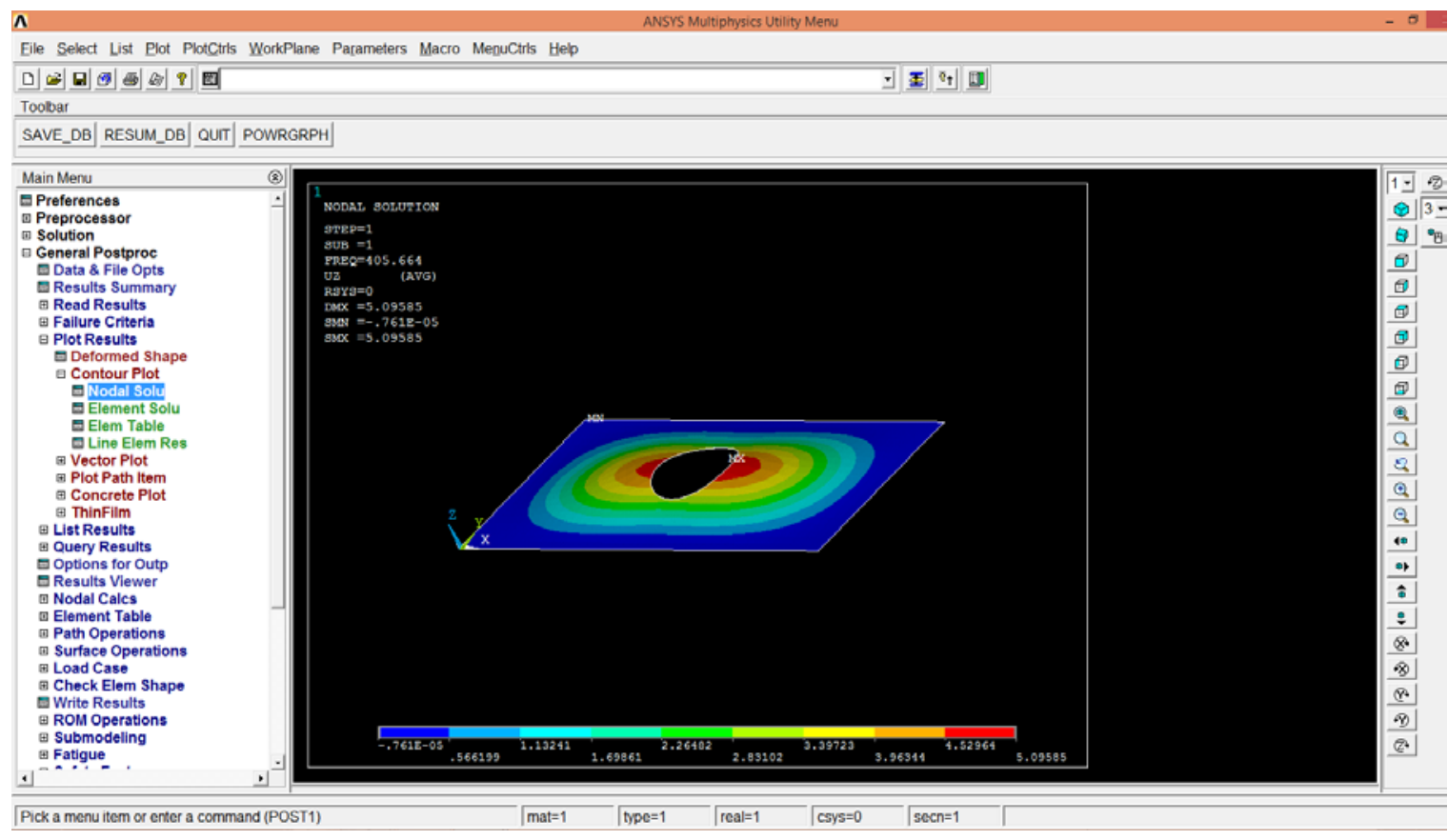

Figure 8: Plate with circular hole CCCC condition

\section{RESULTS AND DISCUSSIONS}

\section{Experimentation and validation:}

The laminates prepared were subjected to dynamic analysis and the natural frequencies were found out. The same are presented for first three modes. Finite element analysis for the various laminates of different boundary conditions was carried out and is as shown in Table 2. From Table 2 one can infer the various frequency values which helps in designing of structures and also for damping studies

The bare plate shows reduced natural frequency when the laminate is under CFCF condition as compared to CCCC condition in $1^{\text {st }}$ mode, which reveals that freeness in the system reduces the natural frequency of the system. The result holds good for remaining two modes. These results are in agreement with results from FEM approach. It can also be seen that there is a substantial increase in natural frequency of laminates with cutouts as compared to that of bare plate. However, there is a little variation in natural frequency among circular and rectangular cutouts. The calculated values of damping for various boundary conditions are as shown in Table 3. It can be seen clearly from the graphs as in Figure 9 that composite plates with rectangular cutouts have a higher damping followed by composite plate with circular hole. It can also be seen from Figure 9 that composite bare plate has got least damping as compared to plate with cutouts.

Table 2: Comparison of natural frequencies from experiment and FEM method

\begin{tabular}{llllllll}
\hline Plates & Boundary & \multicolumn{2}{l}{$1^{\text {st }}$ Mode } & \multicolumn{2}{l}{$2^{\text {nd }}$ Mode } & \multicolumn{2}{l}{$3^{\text {rd }}$ Mode } \\
& Condition & Frequency $(\mathrm{Hz})$ & Frequency $(\mathrm{Hz})$ & Frequency $(\mathrm{Hz})$ \\
\hline \multirow{3}{*}{ Bare Plate } & & FEM & EXP & FEM & EXP & FEM & EXP \\
& CCCC & 293 & 310 & 438 & 615 & 664 & 730 \\
& CCCF & 251 & 250 & 334 & 490 & 490 & 730 \\
Plate with Circular Hole & CFCF & 240 & 225 & 276 & 460 & 375 & 700 \\
& CCCC & 405 & 390 & 468 & 440 & 755 & 730 \\
\multirow{5}{*}{ Plate with Rectangular cutout } & CCCF & 324 & 315 & 427 & 410 & 517 & 490 \\
& CFCF & 320 & 315 & 331 & 350 & 470 & 490 \\
& CCCC & 402 & 260 & 460 & 520 & 750 & 760 \\
& CCCF & 323 & 200 & 421 & 420 & 510 & 625 \\
& CFCF & 318 & 200 & 329 & 380 & 467 & 620 \\
\hline
\end{tabular}


Table 3: Damping Ratio from Experiment

\begin{tabular}{lccc}
\hline Plate & \multicolumn{3}{c}{ Boundary condition } \\
\cline { 2 - 4 } & CCCC & CCCF & CFCF \\
\hline Bare Plate & 0.012 & 0.016 & 0.025 \\
Plate with circular hole & 0.015 & 0.020 & 0.032 \\
Plate with rectangular cutout & 0.019 & 0.025 & 0.05 \\
\hline
\end{tabular}

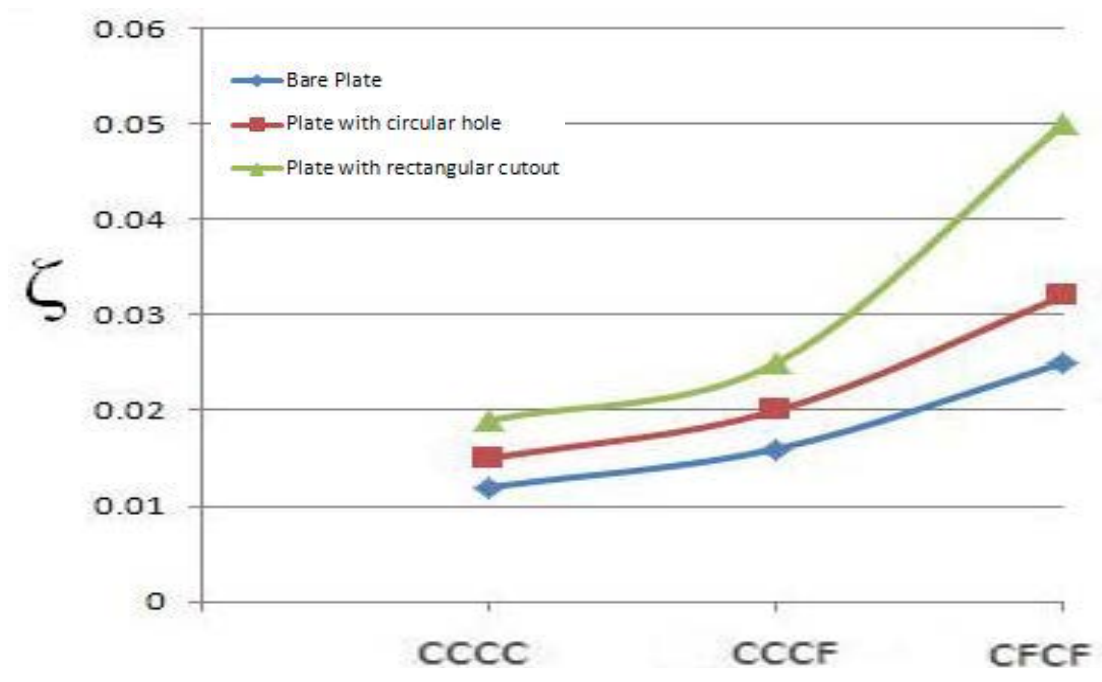

Figure 9: Comparison of damping factor of various composite laminates

It can be seen from the Figure 9 that there is an increase in damping when the configuration changes from CCCC to CFCF for plates with different geometries. The plate constrained in all directions has less damping compared to that of plate, which is constrained in two directions. It can also be observed that the plate with cutout have higher damping compared to bare plate. When geometry of the cutout is considered, there is an increase in damping in rectangular cutout followed by circular cutout and bare plate. The damping could be increased with increased size of cut out.

\section{CONCLUSIONS}

The structural glass fibre reinforced plastic laminates have shown that freeness in the system reduced the natural frequency of the system for bare plate and plates with cutout in all the three modes. If it is intended to increase the natural frequency, one can make cutout in the laminate. It is also seen that the type of cutout whether circular or rectangular has a little impact on natural frequency. Damping is highest in plate with rectangular cutout as compared to plate with circular hole and bare plate for all the types of configuration. It is evident that the damping is less in CCCC configuration for all the three types of plates and increases with increase in freeness of the system as in CFCF configuration. Damping contributes in attenuation of structural vibration mainly at natural frequencies. The freeness in the system reduces.

\section{Scope for Future work}

Laminates can be fabricated using other types of reinforcements and hybrid composites could as well be produced and the same analysis can be carried out on those laminates. Various resin systems can be used to fabricate the polymer composites such as polyester, polyether ether ketone etc.

\section{Acknowledgment}

This research was funded by T.E.Q.I.P-II. The authors are grateful to those who contributed directly and indirectly in producing this paper. 


\section{REFERENCES}

1. Singiresu, S. R. (2011). Mechanical Vibrations, Upper Saddle River, Pearson Education, Inc, Fifth Edition, 3-7.

2. Diveyev, B., Smolskyy, A. \& Sukhorolskyy, M. (2008). Dynamic rigidity and loss factor prediction for composite layered panel. Archives of Materials Science and Engineering, 31(1), 2008, 45-48.

3. Igor, S., \& Moshe, E. (2016). Semi-analytical modeling of cutouts in rectangular plates with variable thicknessFree vibration analysis. Applied Mathematical Modelling, 1-18.

4. David, T. H. (2013). Effects of constraint, circular cut out and in-plane loading on vibration of rectangular plates. International Journal of Mechanical Sciences, 68, 114-124.

5. Liu, X. \& Banerjee, J. R. (2016). Free vibration analysis for plates with arbitrary boundary conditions using a novel spectral-dynamic stiffness method. Computers and Structures, 164, 108-126.

6. Ivo, S., Marko, T., Nikola, V. \& Neven, H. (2015). An approximate analytical procedure for natural vibration analysis of free rectangular plate. Thin-Walled Structures, 95, 101-114.

7. Rui, L., BoWang, Gang, L. \& Bin T. (2016). Hamiltonian system-based analytic modeling of the free rectangular thin plates' free vibration, Applied Mathematical Modelling, 40, 984-992.

8. Xing, Y. F. \& Xu, T. F. (2013). Solution methods of exact solutions for free vibration of rectangular orthotropic thin plates with classical boundary conditions, Composite Structures, 104, 187-195.

9. Ramu, I., \& Mohanty, S. C. (2012). Study on free vibration analysis of rectangular plate structures using finite element method. Procedia engineering, 38, 2758-2766.

10. Eduard, V., Theodor, K. (2001). Thin Plates and Shells. New York Basel, Marcel Dekker, Inc. 